\title{
Erratum to: Reduction of pulmonary toxicity of metal oxide nanoparticles by phosphonate-based surface passivation
}

Xiaoming $\mathrm{Cai}^{1 \dagger}$, Anson Lee ${ }^{5+}$, Zhaoxia $\mathrm{Ji}^{4}$, Cynthia Huang ${ }^{6}$, Chong Hyun Chang ${ }^{4}$, Xiang Wang ${ }^{4}$, Yu-Pei Liao ${ }^{3}$, Tian $\mathrm{Xia}^{3,4^{*}}$ and Ruibin $\mathrm{Li}^{2^{*}}$

\section{Erratum}

After the publication of this work [1] it was noticed that Acknowledgement statement was incorrect. The original statement reads: This work was supported by the grant from the National Natural Science Foundation of China (No. 31671032; 31,570,899), and a project funded by the Priority Academic Program Development of Jiangsu Higher Education Institutions (PAPD). R.L. is supported by the recruitment program of Global Youth Experts of China. However the correct Acknowledgement statement should read as:
Received: 22 August 2017 Accepted: 22 August 2017

Published online: 29 August 2017

\section{Reference}

1. Cai X, Lee A, Ji Z, Huang C, Chang CH, Wang X, et al. Reduction of pulmonary toxicity of metal oxide nanoparticles by phosphonate-based surface passivation. Part Fibre Toxicol. 2017;14:13.

\begin{abstract}
Acknowledgements
This work was supported by the grant from the National Natural Science Foundation of China (No. 31671032; 31570899), and a project funded by the Priority Academic Program Development of Jiangsu Higher Education Institutions (PAPD). This study is also supported by the National Science Foundation and the Environmental Protection Agency under Award No. DBI-1266377, and U.S. Public Health Service Grant, R01 ES016746. R.L. is supported by the recruitment program of Global Youth Experts of China.
\end{abstract}

The correction to the Acknowledgements does not affect any of the experimental results, discussions or conclusions reported in this paper.

\footnotetext{
Author details

${ }^{1}$ Center for Genetic Epidemiology and Genomics, School of Public Health, Jiangsu Key Laboratory of Preventive and Translational Medicine for Geriatric Diseases, Medical College of Soochow University, Suzhou 215123, China. ${ }^{2}$ School for Radiological and Interdisciplinary Sciences (RAD-X), Collaborative Innovation Center of Radiation Medicine of Jiangsu Higher Education Institutions, Jiangsu Provincial Key Laboratory of Radiation Medicine and Protection, Soochow University, Suzhou 215123, China. ${ }^{3}$ Department of Medicine, University of California, Los Angeles, CA 90095, USA. ${ }^{4}$ California NanoSystems Institute, University of California, Los Angeles, CA 90095, USA. ${ }^{5}$ Department of Chemical and Biomolecular Engineering, University of California, Los Angeles, CA 90095, USA. ${ }^{6}$ Department of Microbiology, Immunology, and Molecular Genetics, University of California, Los Angeles, CA 90095, USA.

* Correspondence: txia@ucla.edu; liruibin@suda.edu.cn

${ }^{\dagger}$ Equal contributors

${ }^{3}$ Department of Medicine, University of California, Los Angeles, CA 90095, USA

${ }^{2}$ School for Radiological and Interdisciplinary Sciences (RAD-X), Collaborative Innovation Center of Radiation Medicine of Jiangsu Higher Education Institutions, Jiangsu Provincial Key Laboratory of Radiation Medicine and Protection, Soochow University, Suzhou 215123, China
}

(c) The Author(s). 2017 Open Access This article is distributed under the terms of the Creative Commons Attribution 4.0 International License (http://creativecommons.org/licenses/by/4.0/), which permits unrestricted use, distribution, and reproduction in any medium, provided you give appropriate credit to the original author(s) and the source, provide a link to the Creative Commons license, and indicate if changes were made. The Creative Commons Public Domain Dedication waiver (http://creativecommons.org/publicdomain/zero/1.0/) applies to the data made available in this article, unless otherwise stated. 